\title{
Formulation and Evaluation of Pregabalin Loaded Eudragit S100 Nanoparticles
}

\section{B. Senthilnathan ${ }^{1 *}$, A.Maheswaran ${ }^{1}$, K. Gopalasatheeskumar', K. Masilamani', Raihana Z Edros ${ }^{2}$}

${ }^{1}$ Jaya College of Paramedical sciences, College of Pharmacy, Thiruninravur, Chennai 602024 Tamilnadu, India.

${ }^{2}$ Department of Pharmaceutical Engineering, University Malaysia Pahang, Lebuhraya Tun Razak, 26300 Gambang, Kuantan, Pahang Darul Makmur, Malaysia

sen03mpharm@gmail.com

\begin{abstract}
In this work, polymeric nanoparticles containing Pregabalin was prepared and optimized the ideal concentration of polymer based on its in vitro release profile for a period of 24hrs. The nanoparticles were prepared by solvent displacement method using various concentrations of Eudragit S100 (EPNP1-EPNP5). The prepared nanoparticles were characterized for its particle size, zeta potential, drug content, entrapment efficiency and invitro drug release profile. The preformulation study results confirmed the compatibility between the drug and other excipients used in the formulation. The optimized formulation was selected based on its particle size, entrapment efficiency and in vitro drug release profile. The formulation which contains 300mg of Eudragit S100 (EPNP5) was selected as optimized concentration for the controlled release of Pregabalin for a period of $24 \mathrm{hrs}$.
\end{abstract}

Index Terms- Pregabalin, Eudragit S100, Polymeric nanoparticles, Zeta potential, Encapsulation efficiency.

\section{INTRODUCTION}

The aim of the present work is to develop a controlled release formulation for an antiepileptic drug Pregabalin which is considered as one of the primary drug used in the treatment of neuropathic pain and epilepsy. [1]

Pregabalin on continuous usage $(150-600 \mathrm{mg} /$ day $)$, is reported to cause severe adverse effects including dizziness, drowsiness, blurred vision, increased appetite, euphoria, constipation, vomiting, erectile dysfunction etc and it is required to be taken three times a day.[2-5] These biological situation demand the formulation of Pregabalin loaded Eudragit S100 nanoparticles to achieve controlled release for a period of $24 \mathrm{hrs}$ which in turn reduces the complications of dose size, dosing frequency, adverse effects and improves better patient compliance.

The biological half-life of Pregabalin is 6 hrs, it needs to be taken frequently in order to maintain the steady state blood level concentration. This can be overcome by formulation of controlled release nanoparticles of Pregabalin.

To achieve the objectives the plan is executed to formulate controlled release Pregabalin nanoparticles using Eudragit S100.

II. MATERIALS AND METHODS 


\subsection{Materials Used}

Pregabalin , Eudragit S100 and Poloxamer were purchased from Sigma Aldrich. Ethanol was purchased from Chemika-Biochemika-Reagents, Mumbai. All other chemicals and reagents used were of analytical grade.

\subsection{Methods}

\subsubsection{Preparation of Pregabalin loaded Eudragit S100 nanoparticles}

Solvent displacement method was adopted for the preparation of Pregabalin loaded nanoparticles using various concentration of Eudragit S100 as polymer.[6,7,8] Eudragit S100 in various concentrations were dissolved with $20 \mathrm{ml}$ of ethanol in a beaker. Pregabalin $100 \mathrm{mg}$ was dissolved in a $25 \mathrm{ml}$ of distilled water. The drug solution was then injected to the above polymeric solution under magnetic stirring. The solution was stirred up to $2 \mathrm{hrs}$ until end point is attained. The water and ethanol was evaporated under reduced pressure and final volume is adjusted to $50 \mathrm{ml}$. Several batches namely (EPNP1, EPNP2, EPNP3, EPNP4, EPNP5) were formulated by changing the drug and polymeric ratio and the effect of polymer concentration on the encapsulation efficiency, particle size and in vitro drug release were studied.

Table 1: Formula used for the preparation of Pregabalin loaded Eudragit S100 nanoparticles

\begin{tabular}{|c|c|c|c|c|c|c|}
\hline S.No & Trials & $\begin{array}{c}\text { Pregabalin } \\
(\mathbf{m g})\end{array}$ & $\begin{array}{c}\text { Eudragit S100 } \\
(\mathbf{m g})\end{array}$ & $\begin{array}{c}\text { Poloxamer } \\
(\mathbf{m g})\end{array}$ & $\begin{array}{c}\text { Ethanol } \\
(\mathbf{m l})\end{array}$ & $\begin{array}{c}\text { Purified } \\
\text { Water (ml) }\end{array}$ \\
\hline 1. & EPNP1 & 100 & 100 & 200 & 20 & 20 \\
\hline 2. & EPNP2 & 100 & 150 & 200 & 20 & 20 \\
\hline 3. & EPNP3 & 100 & 200 & 200 & 20 & 20 \\
\hline 4. & EPNP4 & 100 & 250 & 200 & 20 & 20 \\
\hline 5. & EPNP5 & 100 & 300 & 200 & 20 & 20 \\
\hline
\end{tabular}

\subsubsection{Characterization Studies}

\subsubsection{Particle size and Surface charge}

Surface charge is important in adhesion and interaction of particle with cells.[7] The zeta- potential is used to measure the cell surface charge density. It can be measured using Malvern zeta sizer. The prepared nanoparticles were evaluated for their particle size and surface charge by photon correlation spectroscopy (PCS) using zetasizer. [9]

\subsubsection{Drug content}

\subsection{Standard Preparation}

$100 \mathrm{mg}$ of Pregabalin standard was weighed accurately and transferred into a $25 \mathrm{ml}$ volumetric standard flask and dissolved with $5 \mathrm{ml}$ of $\mathrm{pH} 6.8$ phosphate buffer. $1 \mathrm{ml}$ of this solution was diluted with $25 \mathrm{ml}$ with $\mathrm{pH} 6.8$ phosphate buffer.

\subsection{Sample Preparation}

Weighed accurately Pregabalin nanoparticles equivalent to 100mg of Pregabalin and transferred in to a $25 \mathrm{ml}$ standard flask. The sample was dissolved with $5 \mathrm{ml}$ of pH 6.8 phosphate buffer and diluted to $25 \mathrm{ml}$ with $\mathrm{pH} 6.8$ phosphate buffer. $1 \mathrm{ml}$ of this solution was diluted to $25 \mathrm{ml}$ with buffer solution. 
Then the standard and sample absorbance was measured at $210 \mathrm{~nm}$ using a UV-Visible spectrophotometer. The percentage of drug content was calculated.

Drug content $(\%)=\frac{\text { Weight of drug in nanoparticles X } 100}{\text { Weight of nanoparticles }}$

\subsubsection{Entrapment Efficiency}

The drug loaded nanoparticles were exposed to centrifugation at $15000 \mathrm{rpm}$ for $30 \mathrm{~min}$.[10, 11] The supernatant liquid was separated and $1 \mathrm{ml}$ of this solution was diluted with water and the absorbance was measured at $210 \mathrm{~nm}$. The amount of Pregabalin un-entrapped in the supernatant was calculated. The amount of Pregabalin entrapped was determined by subtracting amount of free un-entrapped Pregabalin from the total amount of Pregabalin taken for the preparation.

The formula used to calculate entrapment efficiency was given below [12]

Drug entrapment $(\%)=\frac{\text { mass of drug in nanoparticles } \mathrm{x} 100}{\text { mass of drug used in formulation }}$

\subsubsection{In vitro release}

In vitro release studies were performed for $24 \mathrm{hrs}$ by using the Franz diffusion cell. [13-16] The prepared Pregabalin nanoparticles formulations equivalent to $100 \mathrm{mg}$ of Pregabalin were placed inside a dialysis membrane and immersed in $\mathrm{pH} 6.8$ phosphate buffer. At predetermined time intervals the sample was withdrawn and the amount of Pregabalin released was determined by measuring the absorbance at $210 \mathrm{~nm}$ using a UV-Visible spectrophotometer. From the absorbance values the cumulative percentage drug release was calculated.

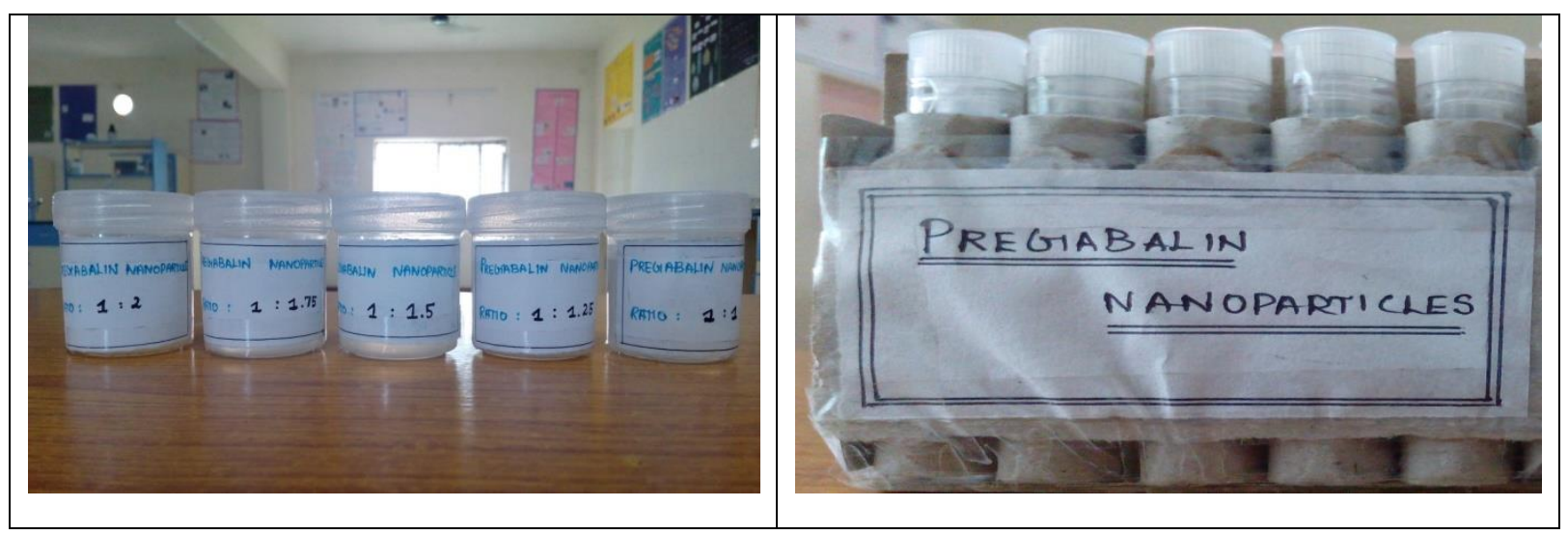

Figure.1-2. pregabalin loaded eudragit s 100 nanoparticles (EPNP1-EPNP5)

\section{Result and Discussion}

\subsection{Drug content and entrapment efficiency}

Particle size and entrapment efficiency of the Pregabalin nanoparticles (EPNP1- EPNP5) were increased with increasing Eudragit S100 concentration.[12] This may be due to high amount of availability of Eudragit S100 to encapsulate the drug, upon increasing the Eudragit S100 content, number of layers coated the drug was increased, this resulted in increased particle size and entrapment efficiency. Further increase in the Eudragit S100 concentration (EPNP5), there is no much increase in the entrapment efficiency due to the availability of the drug to be incorporated is low which is not enough for further encapsulation of drug by Eudragit S100. 
INTERNATIONAL JOURNAL OF ENGINEERING TECHNOLOGY AND SCIENCES (IJETS) Vol.6 (1) Dec 2016 DOI: http://dx.doi.org/10.15282/ijets.6.2016.1.9.1059

Table 2: Drug content and entrapment efficiency Particle size and zeta potential of Pregabalin nanoparticles.

\begin{tabular}{|l|c|c|c|c|}
\hline Trials & $\begin{array}{c}\text { Zeta potential } \\
(\mathbf{m V})\end{array}$ & $\begin{array}{c}\text { Particle size } \\
(\mathbf{n m})\end{array}$ & $\begin{array}{c}\text { Entrapment Efficiency } \\
(\boldsymbol{\%})\end{array}$ & $\begin{array}{c}\text { Drug Content } \\
(\boldsymbol{\%})\end{array}$ \\
\hline EPNP1 & 4.75 & 187.0 & $72.46 \pm 0.24$ & $99.67 \pm 0.08$ \\
\hline EPNP2 & 10.6 & 663.4 & $77.89 \pm 0.08$ & $99.56 \pm 0.24$ \\
\hline EPNP3 & 6.33 & 129.8 & $86.47 \pm 0.09$ & $99.38 \pm 0.31$ \\
\hline EPNP4 & 1.73 & 139.7 & $90.83 \pm 0.15$ & $99.62 \pm 0.27$ \\
\hline EPNP5 & 0.277 & 117.3 & $95.07 \pm 0.45$ & $99.71 \pm 0.19$ \\
\hline
\end{tabular}

Table 3: In vitro release studies of Pregabalin nanoparticles

\begin{tabular}{|c|c|c|c|c|c|c|}
\hline \multirow[t]{2}{*}{ S.NO } & \multirow{2}{*}{$\begin{array}{l}\text { TIME } \\
\text { (Hrs) }\end{array}$} & \multicolumn{5}{|c|}{ \% CUMULATIVE DRUG RELEASE } \\
\hline & & EPNP1 & EPNP2 & EPNP3 & EPNP4 & EPNP5 \\
\hline 1 & 0.5 & $25.56 \pm 0.08$ & $22.46 \pm 0.09$ & $18.45 \pm 0.45$ & $13.37 \pm 0.64$ & $11.38 \pm 0.31$ \\
\hline 2 & 1 & $43.67 \pm 0.34$ & $40.58 \pm 0.31$ & $35.53 \pm 0.27$ & $30.75 \pm 0.15$ & $22.45 \pm 0.27$ \\
\hline 3 & 6 & $75.57 \pm 0.12$ & $71.79 \pm 0.19$ & $67.85 \pm 0.76$ & $61.44 \pm 0.40$ & $54.29 \pm 0.38$ \\
\hline 4 & 12 & $98.54 \pm 0.34$ & $93.72 \pm 0.38$ & $81.36 \pm 0.28$ & $73.65 \pm 0.29$ & $68.76 \pm 0.17$ \\
\hline 5 & 16 & $98.51 \pm 0.27$ & $98.57 \pm 0.19$ & $92.49 \pm 0.38$ & $85.73 \pm 0.17$ & $80.47 \pm 0.28$ \\
\hline 6 & 20 & $98.48 \pm 0.55$ & $98.54 \pm 0.17$ & $98.56 \pm 0.11$ & $93.42 \pm 0.53$ & $91.39 \pm 0.26$ \\
\hline 7 & 24 & $98.47 \pm 0.32$ & $98.52 \pm 0.21$ & $98.55 \pm \quad 0.28$ & $98.57 \pm 0.64$ & $99.58 \pm 0.08$ \\
\hline & & & & & & \\
\hline
\end{tabular}

\subsection{In- vitro drug release}

From the in vitro drug release study results, the maximum percentage drug release $(99.58 \pm 0.08)$ at the end of 24hrs was observed with trial EPNP5 which contains $300 \mathrm{mg}$ of Eudragit S100.

Below $300 \mathrm{mg}$ of Eudragit S100 concentration as in the case of trials EPNP1, EPNP 2,EPNP 3 and EPNP 4, the maximum percentage drug release 98.54 $\pm 0.34,98.57 \pm 0.19,98.56 \pm 0.11$ and $98.57 \pm 0.64$ were obtained at the end of $12,16,20$ and $24 \mathrm{hrs}$ respectively which was not desirable. 
INTERNATIONAL JOURNAL OF ENGINEERING TECHNOLOGY AND SCIENCES (IJETS) Vol.6 (1) Dec 2016 DOI: http://dx.doi.org/10.15282/ijets.6.2016.1.9.1059

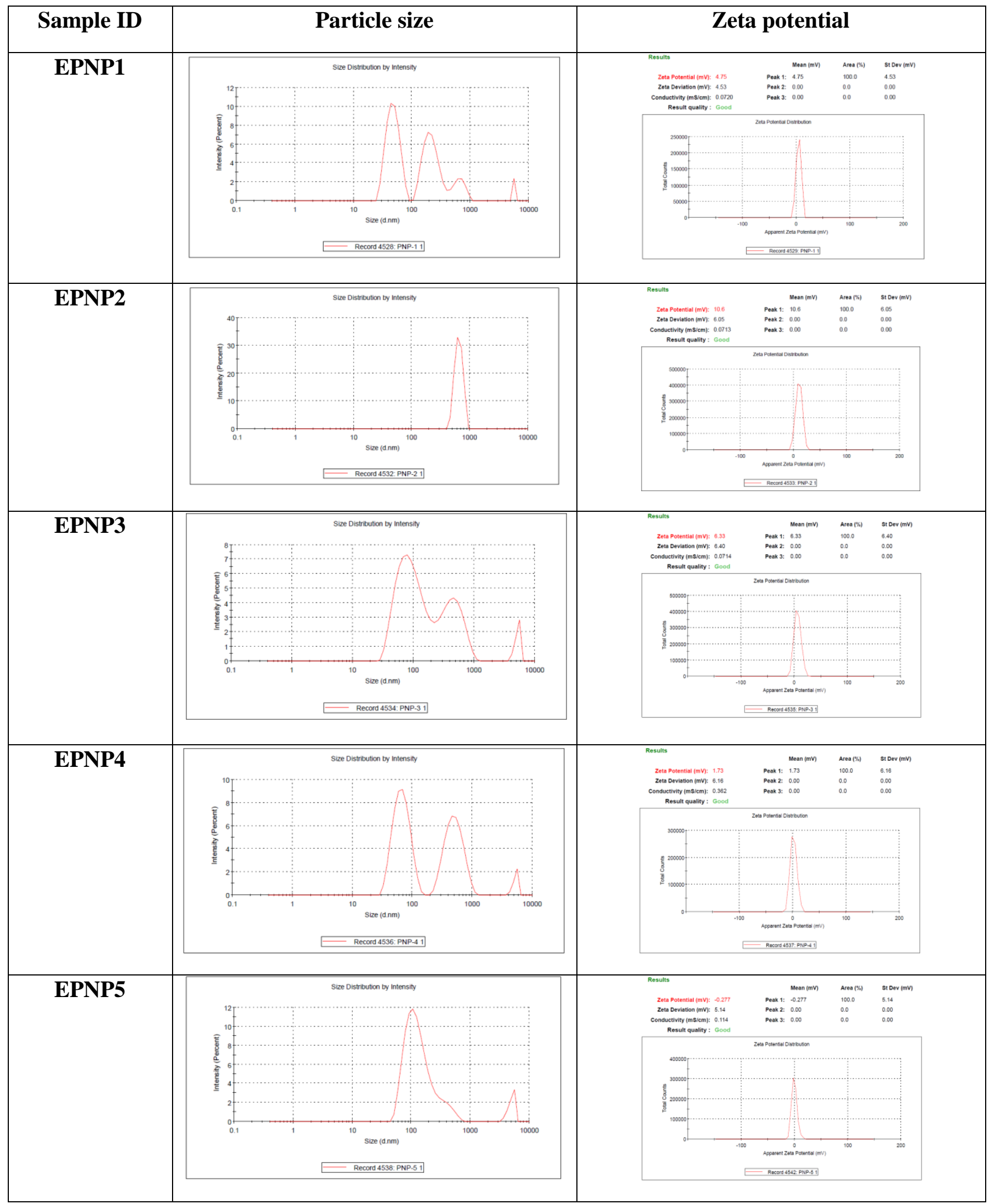

Figure.3-12. Particle size and Zeta potential 
Beyond $250 \mathrm{mg}$ of Eudragit S100 concentration, reduction in drug release was observed in the case of trial EPNP5. The maximum percentage drug release for EPNP5 was found to be $99.58 \pm 0.08$ at the end of $24 \mathrm{hrs}$ were obtained.

From the in vitro drug release data for EPNP1- EPNP5, it was observed that increase in Eudragit S100 concentration delays the drug release due to increased particle size and reduced surface area available for drug release.

From all the formulations, EPNP5 was selected as best formulation due to its ideal particle size $(\mathrm{nm})$, high entrapment efficiency $(95.07 \pm 0.45 \%)$ and desirable drug release $(99.58 \pm 0.08 \%$ at $24 \mathrm{~h})$.

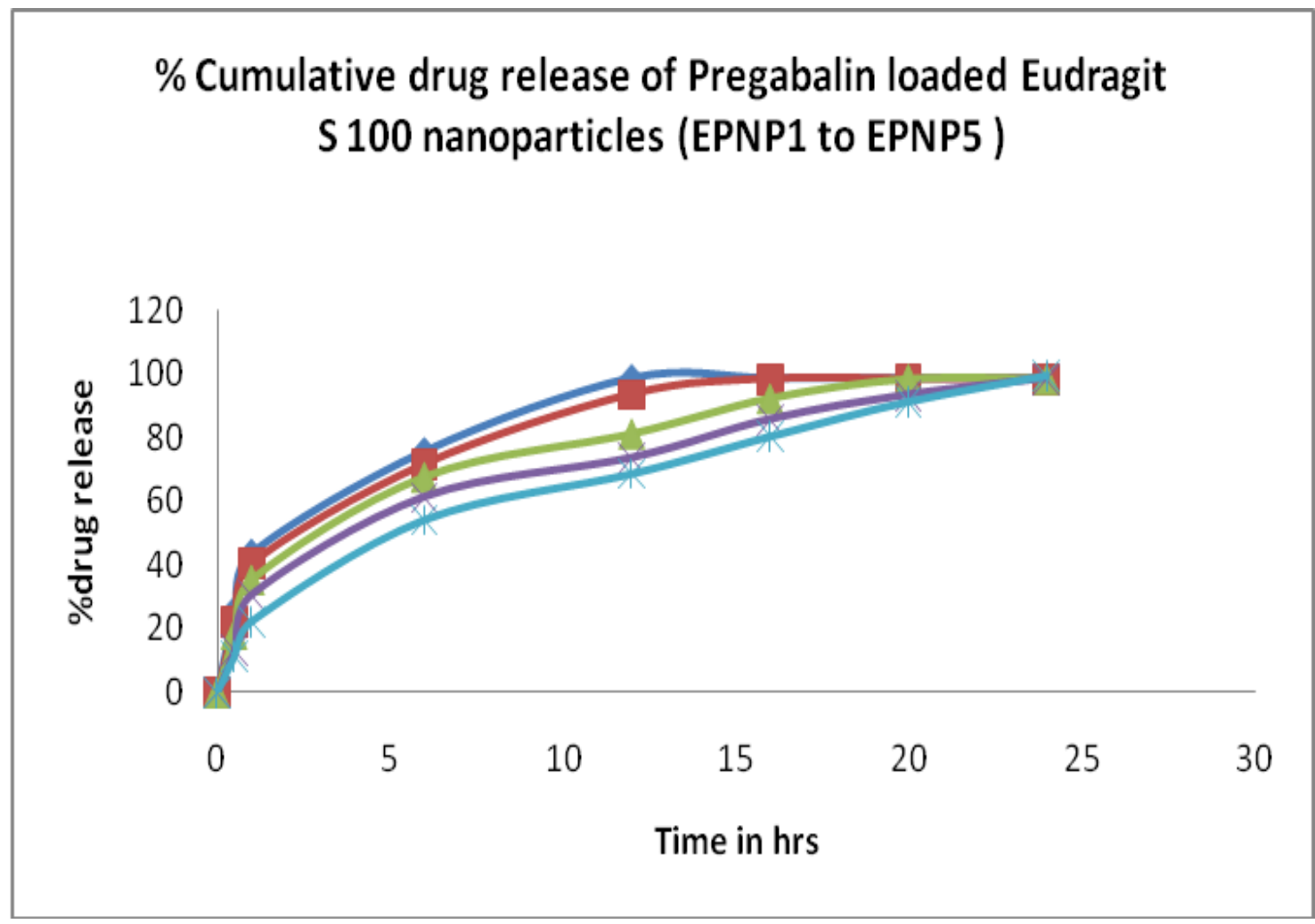

Fig. $13 \%$ Cumulative drug release of Pregabalin loaded Eudragit S 100 nanoparticles (EPNP1 to EPNP5)

\section{CONCLUSION}

In the present study Eudragit S100 nanoparticles containing Pregabalin was prepared by solvent displacement technique. The effect of increase in polymer concentration in various parameters like particle size, zeta potential, entrapment efficiency and in vitro release profile were studied. The results showed that the in vitro drug release for EPNP1, EPNP2, EPNP3, EPNP4 and EPNP5 were found to be $98.47 \pm 0.32,98.52 \pm 0.21,98.55 \pm 0.28,98.57 \pm 0.64$ and $99.58 \pm 0.08$ respectively. Based on the $i n$ vitro drug release profile of Pregabalin nanoparticles formulations (EPNP1-EPNP5) EPNP5 was selected as the best formulation in which the particle size was $117.3 \mathrm{~nm}$ and drug:polymer in the ratio of 1:3 (Pregabalin 100mg:300mg of Eudragit S100). The in vitro \% drug release of EPNP5 formulation was $99.58 \pm 0.08$ and it was found to be suitable formulation for the epileptic patient to manage the symptoms of epilepsy. Hence it can be concluded that the newly formulated Pregabalin loaded Eudragit S 100 nanoparticles may be ideal and effective to control the epilepsy by allowing the drug to release continuously for $24 \mathrm{hrs}$. 


\section{References}

1. Nagarajan E, Shanmugasundaram P, Ravichandiran V, Vijayalakshmi A, Senthilnathan B, Masilamani K. "Development and Evaluation of Chitosan Based Polymeric Nanoparticles of an Antiulcer Drug Lansoprazole", Journal of Applied Pharmaceutical Science 5 (04):20-25.2015.

2. Anusha R, Somasundaram I, Ravichandiran V, Kausalya J, Joseprakash, D Senthilnathan B, "Effect of Cholesterol and Surfactant Concentration On Solid Lipid Nanosuspension Of Ropinirole Hydrochloride", Journal of Pharmacy Research" 4(6),1606-1609.2011.

3. Senthilnathan B, Ameerkhan H, Aswini S, Abirami M, Bharath T, Ajithkumar T and Maheswaran A "Review on various approaches on preparation, Characterisation and applications of polymeric Nanoparticles”, World Journal of Pharmaceutical Research 4(6):645-663.2015.

4. Karpagavalli L, Senthilnathan B, Maheswaran A, Narayanan N. "Formulation and evaluation of Aceclofenac nanocapsules", Invent Rapid:NDDS 4:1-6.2015.

5. ChizobaEkezie FD, Suneetha J, Uma Maheswari WK, Prasad TNVKV. "Zinc nanoparticulated bitter gourd extract: in-vitro anti-diabetic efficacy", International Journal of Science and Nature, 6(4):687-692.2015.

6. Antal I, Kubovcikova M, Zavisova V, Koneracka M, Pechanova O, Barta A. "Magnetic poly(D,L-lactide) nanoparticles loaded with aliskiren: a promising tool for hypertension treatment", Journal of Magnetism and Magnetic Materials 380 280-284.2015.

7. Fargnoli AS, Mu A, Katz MG, Williams RD , Margulies KB, Weiner DB, Yang S Bridges CR. "Anti-inflammatory loaded poly-lactic glycolic acid nanoparticle formulations to enhance myocardial gene transfer: an in-vitro assessment of a drug/gene combination therapeutic approach for direct injection", Journal of Translational Medicine 2014, 12:171.2014.

8. Reddy AS, Sailaja AK "Preparation characterization of aspirin loaded ethylcellulose nanoparticles", WJPPS. 3(6):1781-1793.2014.

9. Sagar SJ, Aparna V B "Formulation characterization of aspirin loaded ethylcellulose nanoparticles". WJPPS 3(6):1781-1793.2014.

10. Kumar GP, Razdan BK, Meenakshi B "Formulation and evaluation of nanoparticles containing aetemisinin HCL", International Journal of Research and Development in Pharmacy and Life Sciences, 3(2):925-934.2014.

11. Lokhande AK, Sathyendra, Kulkarni R ,"Formulation and evaluation of Glipizide loaded nanoparticles", IJPPS, 05(04):147-151.2013.

12. Srinivas P, Pragna S. "Formulation and evaluation of moxifloxacin HCL ocular nanoparticles" IJND. 3(2):105-113.2012.

13. Naik JB, Mokale VJ "Formulation and evaluation of Repaglimide nanoparticles as sustained release carriers", IJPS, 1(5):259-266.2012.

14. Kesarkar R, Oza G, Pandey S, Dahake R, Mukherjee S, Chowdhary A, Sharon M "Gold nanoparticles: effective as both entry inhibitors and virus neutralizing agents against HIV", Scholars Research Library, J. Microbiol. Biotech. Res., 2 (2):276-283.2012.

15. Choi SW, Kim JH "Design of surface-modified poly (d,1-lactide-co-glycolide) nanoparticles for targeted drug delivery to bone", J Control Release, 122:24-30.2007.

Kawashima Y, "Nanoparticulate systems for improved drug delivery", Adv Drug Deliv Rev 47:12.2001 . 